\title{
Severe Life Events and Chronic Adversities as Antecedents to Anxiety in Children: A Matched Control Study
}

\author{
Jennifer L. Allen • Ronald M. Rapee $\cdot$ Seija Sandberg
}

Published online: 3 June 2008

(C) Springer Science + Business Media, LLC 2008

\begin{abstract}
The present study compared the number of severe life events and chronic adversities as reported retrospectively by mothers of children with an anxiety disorder $(n=39)$ prior to the onset of their most recent episode, with controls $(n=39)$ matched for age and sex. The parent version of the Psychosocial Assessment of Childhood Experiences (PACE) was used to assess chronic adversities (long-term experiences with negative impact on child) and severe life events (discrete life events with high long-term threat). A significantly greater number of severe life events and chronic adversities were assessed prior to onset for anxious children compared to controls. The finding for severe life events held regardless of whether impact ratings were assigned by mothers or a panel of independent raters, suggesting the findings reflect actual as opposed to perceived differences. Results suggest that both discrete
\end{abstract}

\footnotetext{
J. L. Allen

Clinical Child and Adolescent Psychology Department,

University of Basel,

Missionsstrasse 60/62,

Basel 4055, Switzerland

J. L. Allen $(\square)$

Department of Psychology, Macquarie University,

Sydney, Australia

e-mail: Jennifer.allen@unibas.ch

R. M. Rapee

Centre for Emotional Health, Macquarie University,

Sydney, Australia

S. Sandberg

Department of Mental Health Sciences,

Universtiy College London,

London, United Kingdom
}

and chronic stressors may constitute risk for future episodes of anxiety in children.

Keywords Child anxiety · Etiology · Stress · Life events . Chronic adversity

Theoretical models of anxiety have identified stressful life events as a potential contributing factor to the development and maintenance of anxiety in children (Chorpita and Barlow 1998; Hudson and Rapee 2004; Rapee 2001). At present, these models do not differentiate between the chronicity of different events but appear to assume that stressors are discrete in nature. In contrast, the life events literature distinguishes chronic adversities, which refer to enduring stressors, from life events, which are discrete (time-limited) occurrences with a clearly identifiable onset and ending (Goodyer 2001). Indeed, empirical data have shown that anxious children experience both more chronic adversities and stressful life events than control children (Boer et al. 2002; Eley and Stevenson 2000; Rapee and Szollos 2002). Hence exposure to any form of adverse experience regardless of its duration might be predicted to increase children's vulnerability to anxiety. On the other hand, it has been suggested that chronic adversity in childhood may predict the early onset of anxiety and chronic anxiety disorders, whereas episodic disorders (e.g., depression) may be more likely to be triggered by life events (Phillips et al. 2005). Following from these considerations, examination of both life events and chronic adversities prior to the onset of anxiety will have fundamental implications for current theoretical models.

Distinguishing life events and chronic adversities that precede the onset of clinical episodes from those that occur during such episodes is crucial if a causal link between stressors and the timing of onset is to be determined 
(Goodyer 2001). Several studies have shown that anxious adults report a significantly greater number of life events in the 12 months prior to the onset of their most recent clinical episode in comparison to controls (Blazer et al. 1987; Faravelli 1985; Finlay-Jones and Brown 1981), although the evidence has not been completely consistent (Rapee et al. 1990; Roy-Byrne et al. 1986). In contrast, few studies of this nature have been conducted with anxious children. This is surprising given that as most anxiety disorders take a chronic course, environmental factors important to onset are likely to be those present in childhood (Rapee and Bryant 2008). The limited evidence available indicates that anxious children experience a greater number of life events and some specific chronic adversities in the 12-month period preceding the onset of anxiety in comparison to healthy controls (e.g., Goodyer et al. 1988, 1990).

Although there is some evidence that childhood anxiety is preceded by stressful life events and chronic adversities, several methodological features of these studies limit the value of their findings for models of the development and maintenance of anxiety disorders. First, in two of the studies anxious children were identified by an unstructured psychiatric interview and were obtained from severely disturbed populations attending in-patient and outpatient hospital-based psychiatric clinics (Goodyer et al. 1988, 1990), which is not characteristic of most anxious children. The only study to identify children using a structured psychiatric interview (Gothelf et al. 2004) relied on a child self-report checklist to assess life events and thus failed to provide a more independent determination of the "severity" or impact of stressors. This is crucial given evidence suggesting that the greater number of life events reported by anxious adults compared to controls may reflect perceived rather than actual differences (Rapee et al. 1990).

Investigator-based interviews are widely considered to be the "gold standard" in life events measurement, chiefly due to the inclusion of ratings of contextual threat provided by independent raters. Contextual threat refers to the level of threat caused by the life event to an average child of the same, age, sex and biographical characteristics as those of the child in question (Sandberg et al. 1993). This concept provides an objective yet personalised measure of impact, recognizing the different circumstances of children and the context within surrounding events but avoiding the problem of subjective bias. Studies of life events in children have tended to rely on mother report. However, it has been suggested that mothers of anxious children may also inflate the impact of life events due the influence of their own anxiety (Eley and Stevenson 2000). As yet, no study has compared the number of life events prior to onset calculated on the basis of both maternal and investigator-based ratings of impact in children with a formal diagnosis of an anxiety disorder and healthy controls.
Previous studies with anxious children have either provided no detail as to how onset was defined or assessed (e.g., Gothelf et al. 2004), or have employed a definition based on the appearance of symptoms of anxiety (e.g., Goodyer et al. 1988, 1990). Given the marked overlap between pre-existing temperamental traits and anxiety disorder (e.g., Hudson and Rapee 2004; Pérez-Edgar and Fox 2005; Rapee and Spence 2004), focus on impairment in the child's psychosocial functioning in addition to anxiety symptoms may provide a clearer definition of onset (Rapee and Spence 2004; Sandberg et al. 2001). Such a definition would be more consistent with the requirement in DSM-IV (APA 1994) that diagnosis involves significant life impairment. Finally, a distinction has been made between independent and dependent life events, defined as events that could and could not have been brought about by an individual's own behavior (Sandberg et al. 1993; Sandberg and Rutter 2008). Thus bereavement is an example of an independent event, while conflict with a friend is an example of a dependent event, given that this event is likely to be related, at least in part, to a child's own behavior. In the current study only life events and chronic adversities independent of the child's behavior were included to avoid a confounding of symptoms and events (e.g., Eley and Stevenson 2000).

The current study aimed to determine the number of severe life events and chronic adversities experienced by children with an anxiety disorder prior to the onset of their most recent episode in comparison to control children matched on age and sex. Anxious children were obtained from an anxiety disorders outpatient unit and were diagnosed through structured clinical interview. Furthermore, study was confined to severe life events based on evidence suggesting that child psychopathology is chiefly associated with life events carrying high long-term threat (e.g., Eley and Stevenson 2000; Goodyer et al. 1988, 1990; Sandberg et al. 1993). Determination of events and adversities was made on the basis of a standardized interview that included extensive contextual information, thereby allowing a panel of experts to determine severity independent of the mothers' own ratings. It was hypothesized that compared to matched controls, anxious children would experience a significantly greater number of (a) severe life events on the basis of both maternal and contextual ratings of impact, and (b) chronic adversities in the 12 months preceding the onset of their most recent episode of anxiety. It was also predicted that a significantly greater number of severe life events would be assessed for anxious children on the basis of maternal in comparison to contextual ratings, due to maternal perceptual bias. Significant positive relationships between maternal and child self-reported anxiety symptoms and the number of severe life events and chronic adversities were predicted for the clinical sample. 


\section{Method}

Participants

The clinical sample consisted of 39 children (19 boys and 20 girls) aged 6 to 12 years $(M=9.6$ years, $\mathrm{SD}=1.7)$ and their mothers recruited through the Macquarie University Anxiety Research Unit, Sydney, Australia. Participants were consecutive admissions to our treatment clinic and all families who were approached agreed to participate. Clinical children were required to meet DSM-IV criteria for an anxiety disorder as their primary diagnosis (most interfering), based on a structured clinical interview (see below). Participants were excluded if parents reported that a non-anxiety diagnosis had ever been present prior to the emergence of anxiety symptoms and associated interference for their child. Primary diagnoses were as follows: Generalized Anxiety Disorder 48.7\%, Separation Anxiety Disorder 28.2\%, Specific Phobia 7.7\%, Social Phobia 7.7\% and Obsessive-Compulsive Disorder 7.7\%. Only seven children were in their first episode of anxiety. Comorbidity among the anxiety disorders was common, as $85 \%$ of children met criteria for more than one anxiety disorder. Approximately one-fifth of the sample met criteria for additional non-anxiety comorbid disorders, including depressive disorders $(n=3)$ and externalising disorders $(n=4)$.

The control sample consisted of 39 children (19 boys and 20 girls) aged between 7 and 12 years $(M=9.6$ years, $\mathrm{SD}=1.6)$ and their mothers. Control children were matched to anxious children on sex and age within 4 months of either side of the date of birth of the anxious child ( $M=$ 2.0 months, $S D=1.4$ ). The control sample was recruited via advertisements in school bulletins asking for children who had never seen a mental health professional and their mothers to participate. Control mothers and children received $\$ 50$ in return for completing the questionnaires, interviews and some additional tasks. Mothers and children were included as controls only if the child did not meet DSM-IV criteria for any mental disorders on the basis of a structured clinical interview (see below).

\section{Measures}

The Anxiety Disorders Interview Schedule for DSM-IV: Child and Parent versions (ADIS-C/P: Silverman and Albano 1996) are semi-structured interviews designed to assess anxiety diagnoses and a range of other disorders commonly experienced by children. The ADIS-C/P provides clinician-based combined diagnoses on the basis of child and parent report of symptoms and impairment. The primary diagnosis represents the disorder currently causing the greatest life interference. Additional diagnoses represent all other disorders where DSM-IV criteria are met. Graduate students in clinical psychology or qualified clinical psychologists provided diagnoses following ADIS-C/P administration. The ADIS-C/P has good to excellent test-retest reliability for combined diagnoses (Silverman et al. 2001). Research from our clinic has demonstrated inter-rater agreement of $K=1.00$ for an overall diagnosis of anxiety, and good to excellent inter-rater agreement across the major child anxiety diagnoses, with kappas ranging from 0.68 to 0.93 (Lyneham et al. 2007).

The Spence Children's Anxiety Scale: Child and Parent versions (SCAS-C/P: Spence 1998, 1999) assess anxiety symptoms in children. Both child and parent versions contain 38 items that all load on a single higher order scale, with scores ranging from 0 to 114 . The total scale has good internal consistency (0.92) and 6-month retest reliability (Spence 1998). The SCAS-P shows satisfactory to excellent reliability and is able to differentiate between anxious and non-clinical children (Nauta et al. 2004). Cronbach's alphas for the child and parent SCAS in the current sample were 0.87 and 0.94 .

Further clinical descriptive information was obtained using the Strengths and Difficulties Questionnaire: Child and Parent versions (SDQ-C/P; Goodman 1997), 25-item scales designed to assess symptoms of psychopathology and prosocial behaviour in children. The SDQ consists of five scales: emotional symptoms, conduct problems, hyperactivity, peer problems and prosocial behaviour. The first four scales are summed to yield a total difficulties score, with a range from 0 to 40 . The SDQ has been shown to have satisfactory reliability and validity (Goodman 2001). Cronbach's alphas for the child and parent SDQ total difficulties scores in the current sample were 0.85 and 0.86 .

The Psychosocial Assessment of Childhood Experiences: Parent Version (PACE; Glen et al. 1993; Sandberg et al. $1993)$ is a standardized investigator-based interview designed to assess life events and chronic adversities experienced by children. This interview was based on the principles of the Life Events and Difficulties Schedule (LEDS; Brown and Harris 1978). PACE covers the following areas of the child's life: moves, changes in the household (births, people entering/leaving), separations from family, illnesses, accidents and hospital contacts, family and peer relationships, marital events, witnessing or experiencing a traumatic event, significant achievements and exceptionally good experiences. The principal criteria for inclusion as a life event are that most children of the same age as the participant would find the experience either: (a) threatening, unpleasant or upsetting, or (b) very pleasant, enhancing to self-esteem, or opening up new opportunities. In addition to questions about event occurrence and timing, additional information is elicited about the circumstances surrounding each life event. PACE has been validated for both 12- and 18-month assessment time 
periods (Sandberg et al. 1993, 1998). In order to maximize recall accuracy, the shorter, 12-month timeframe was selected for the present study as mothers were asked to report events that may have occurred at potentially quite some time prior to their interview.

After the completion of the PACE interviews, a panel of trained raters experienced in the administration and scoring of PACE assign ratings of the contextual threat and independence of life events on the basis of consensus agreement amongst panellists. Both panel (contextual) and parent (subjective) ratings of life event impact are assessed using a 4-point scale (0-3), with higher scores indicating greater threat. Severe life events included those rated 2 or more on their long-term threat ( 2 weeks later) for the child. Chronic adversities are conceptualised as the long-term equivalent of discrete life events, for example chronic bullying or parent conflict. Chronic adversities are rated for their objective impact (again on a 4-pt scale) by the panel. An ongoing experience was classified as a chronic adversity if it lasted for a minimum of 4 weeks' duration and was rated as exerting a negative impact on the child (score of at least 1). Panel ratings of the independence of life events are assessed using a 4-point ordinal scale. In this study, dichotomous scores were obtained by combining categories of "totally independent" and "probably independent", and "totally" and "probably behaviour-related". Only life events and chronic adversities that were probably or totally independent, and therefore unlikely to have been caused by a child's behaviour were included in the analyses. Positive events were not examined as no theoretical models have identified them as a factor that contributes to the development of child anxiety.

\section{Procedure}

Mothers who agreed to participate were sent information and consent forms approved by the University Ethics Board to read and sign. Questionnaires were mailed to mothers 1 week before the interviews and returned at the time of the ADIS:C/P. Mothers of anxious children were later interviewed by the first author to ascertain the timing of onset. Onset was defined as the occurrence of the most recent clinical episode of anxiety, requiring the presence of both anxiety symptoms and related clinically significant impairment in the child's psychosocial functioning. Mothers were not informed that they would be interviewed about life events preceding onset until after the timing of onset was established. The interviewer assisted mothers to date onset as precisely as possible using a timeline showing significant dates (e.g., school term times, major holidays) and any personal time landmarks such as birthdays or changes in residence. The average duration of the current anxiety episode was $5 \pm 4$ months (range 1-14 months). Therefore the time-period over which mothers were asked to recall events ranged from 13 to 26 months, with the median duration of recall being 15 months.

Mothers of anxious children were interviewed using PACE to assess life events and chronic adversities in the 12-month period prior to the onset of their child's most recent clinical episode. Mothers of controls were asked about an equivalent time period to the mother of the anxious child they were matched to. That is, a mother of a control child matched to an anxious child whose onset was 6 months prior to their mother's PACE interview would be asked to report on experiences dating from 6 to 18 months prior to the time of their own PACE interview. Hence, the dates reported on by mothers of anxious children and their matched control counterparts were equivalent. During the interview additional events were recorded on the timeline and used as memory cues to further facilitate accurate event dating.

All PACE interviews were conducted by the first author (J.L.A.), who completed extensive training with the third author (S.S.), one of the creators of the PACE interview. Panel meetings were conducted by two panellists, comprised of the first author (J.L.A.) and the third author (S.S.). During these meetings the PACE interviewer (J.L.A.) presented the second panellist (S.S.) with written synopses of reported life events and chronic adversities in chronological order in addition to the biographical details about the child (e.g., age, sex, health, social support, family finances). The second panellist (S.S.) was blind to subjective aspects of the interview, such as individual reactions to event(s) and child diagnostic status. The panel process produced consensus judgements regarding whether the experience qualified as an event, in addition to ratings of contextual threat and independence. In the rare instances where panel members could not agree, the third author (S.S.) made the final decision regarding the rating in recognition of her extensive experience with the panel process. Following standard PACE procedure, decisions regarding whether each reported experience met criteria to be classified as a chronic adversity were made during the panelling process, precluding the assessment of mother impact ratings for chronic adversities.

\section{Results}

\section{Demographics}

Most children had two parents in the home (94\%), although a small percentage came from single-parent families $(6 \%)$. All mothers were the child's primary caregiver. Mothers identified their family's ethnicity as follows: $85 \%$ Australian, 9\% European, 3\% Chinese and 3\% Indian. Examination of household income revealed a predominantly upper class sample (see Table 1). Groups were compared on 
Table 1 Group Differences on Demographic Characteristics and Clinical Measures

\begin{tabular}{lccc}
\hline & Anxious & Control & \multicolumn{1}{l}{$t / \chi^{2}$} \\
\hline Maternal age, $M(\mathrm{SD})$ & 40.1 & 41.9 & 1.502 \\
Number of siblings, $M(\mathrm{SD})$ & 1.5 & 1.7 & 1.055 \\
Australian, \% $(n)$ & $82(32)$ & $87(34)$ & 0.394 \\
Maternal relationship status, & & & \\
\% $(n)$ & & & \\
Married/de facto & $100(40)$ & $87(34)$ & $5.342^{*}$ \\
Single & $0(0)$ & $13(5)$ & \\
Mother completed 12 years & $87(34)$ & $90(35)$ & 0.126 \\
$\quad$ education, \% $(n)$ & & & \\
At least one parent employed & $92(36)$ & $92(36)$ & 0.000 \\
$\quad$ full-time, \% $(n)$ & & & \\
Household income, \% (n) & & & \\
$\quad<40,000$ & $13(5)$ & $5(2)$ & 4.176 \\
\$40,000-\$80,000 & $50(19)$ & $35(13)$ & \\
$>\$ 80,000$ & $37(14)$ & $60(22)$ & \\
SCAS-P, $M(\mathrm{SD})$ & $35.1(15.0)$ & $8.5(6.6)$ & $-10.170^{* *}$ \\
SCAS-C, $M(\mathrm{SD})$ & $36.5(15.4)$ & $13.0(8.4)$ & $-8.349 * *$ \\
SDQ-P, $M(\mathrm{SD})$ & $17.6(4.5)$ & $5.4(4.5)$ & $-12.078^{* *}$ \\
SDQ-C, $M(\mathrm{SD})$ & $18.6(4.8)$ & $8.1(5.7)$ & $-8.830^{* *}$ \\
\hline
\end{tabular}

${ }^{*} p<0.05,{ }^{* *} p<0.001$

demographic variables using two-tailed independent groups two-tailed $t$ tests and chi-squares. Correction of the type 1 error rate was not employed so that differences were not masked by excessive conservatism. There were no significant differences between the clinical and control group for maternal age or number of children's siblings. Groups did not differ with regard to household income, maternal education or whether at least one parent was in full-time paid employment. Significantly more mothers of controls reported being single in comparison to mothers of anxious children. Maternal relationship status was therefore included as a covariate in later analyses to control for differences between samples.

\section{Group Differences on Clinical Measures}

Two-tailed independent samples $t$ tests revealed significant differences between anxious and control children on maternal report of child anxiety and child self-reported anxiety. Means presented in Table 1 show that anxious children scored higher than controls on both the SCAS-C and SCAS-P, indicating greater anxiety. There was also a significant difference between groups on the SDQ-C and SDQ-P, with higher levels of psychopathology reported for clinical children than controls.

Assessment of Severe Life Events and Chronic Adversities

PACE identified a total of 509 life events and 226 chronic adversities. Of the 346 life events judged as independent of the child's behaviour, $90(26 \%)$ were identified as severe on the basis of panel ratings, while mothers identified 53 severe life events (10\%). Of the 177 chronic adversities identified as independent, the vast majority $(90 \%)$ were assessed as exerting a negative impact. Nine life events (including five severe life events) and two chronic adversities were rated as neutral in impact (i.e., neither more positive nor more negative) by the panel due to their complex nature, and therefore excluded from relevant analyses along with their maternal equivalent. Examples of "neutral" events included a child's mother becoming engaged (life event) and an extended stay with relatives prior to moving into a new home (chronic adversity). No events were rated neutral by mothers.

\section{Reliability of Panel Ratings}

Reliability was determined by randomly selecting a subset of participants $(n=20)$ and comparing their panel ratings with those provided by a trained external rater. This rater was presented with the written synopses for this subset and was blind to subjective information including child diagnostic status. The level of absolute agreement between panel ratings and those provided by the external rater was examined using the intraclass correlation coefficient (ICC). ICCs were calculated for the variables used in the analyses, i.e., the number of severe life events and chronic adversities rated as independent from the child's behaviour. ICCs were 0.79 for the number of severe life events and 0.73 for the number of chronic adversities.

The Relationship Between Child Anxiety Symptoms, Severe Life Events and Chronic Adversities

Correlations were calculated between child self-reported anxiety (SCAS-C total scores) and mother report of child anxiety (SCAS-P total scores) and the number of severe life events and chronic adversities separately for the clinical and control groups (see Table 2). There were no significant correlations between the number of severe life events

Table 2 Correlations Between Child Anxiety Symptoms and the Number of Severe Life Events and Chronic Adversities: Anxious and Control Children

\begin{tabular}{lllllll}
\hline & Anxious & & & \multicolumn{2}{c}{ Controls } & \\
\cline { 2 - 3 } \cline { 6 - 7 } \cline { 6 - 7 } & SCAS-P & SCAS-C & & SCAS-P & SCAS-C \\
\hline Severe life events (mother) & 0.20 & 0.30 & & 0.05 & 0.07 \\
Severe life events (panel) & 0.09 & 0.18 & & 0.10 & 0.18 \\
Chronic adversities (panel) & $0.36^{*}$ & $0.39^{*}$ & & 0.24 & 0.23 \\
\hline
\end{tabular}

$* p<0.05$ 
assessed on the basis of panel or maternal ratings and SCAS-C or SCAS-P scores for either the clinical or control group. There were significant moderate positive correlations between SCAS-C and SCAS-P scores and the number of chronic adversities for the clinical sample. In contrast, there were no significant correlations between the number of chronic adversities and SCAS-C or SCAS-P scores for controls.

Comparison of the Number of Severe Life Events Assessed using Panel and Maternal Ratings: Anxious Versus Control Children

A 2(Group: Anxious, Control) $\times 2$ (Rater: Mother, Panel) multivariate analysis of covariance (MANCOVA) was conducted on the number of severe life events assessed for anxious and control children, with maternal relationship status as a covariate ${ }^{1}$. The between-subjects main effect for Group was significant, $F(1,75)=5.387$, partial $\eta^{2}=0.067$, $p=0.023$, with more severe life events reported for anxious children in comparison to controls. There was a significant within-subjects effect of Rater on the number of severe life events, $F(1,75)=15.475$, partial $\eta^{2}=0.171, p<0.001$. Bonferroni follow-up tests of simple effects (critical alpha $p<0.025)$ revealed that more severe life events were assessed overall on the basis of panel ratings in comparison to maternal ratings for both groups, $t(75)=4.194$, $p<0.001 ; M \mathrm{~s}=1.15,0.68$. There were no significant interactions between Group and Rater, $F(1,75)=0.734$, partial $\eta^{2}=0.010, p=0.394$. Adjusted means and standard errors of the number of severe life events for clinical and control samples are presented in Table 3.

\section{Comparison of the Number of Chronic Adversities}

An analysis of covariance (ANCOVA) was conducted to compare the two groups on the number of chronic adversities, including maternal relationship status as a covariate. There was a significant between-subjects main effect for Group, $F(1,77)=5.618$, partial $\eta^{2}=0.070, p=$ 0.020 . PACE assessed a significantly greater number of chronic adversities for anxious compared to control children. Adjusted means and standard errors for the number of chronic adversities assessed in the clinical and control samples are presented in Table 3 .

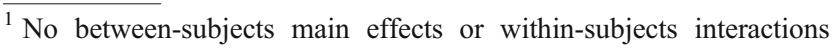
involving the covariate maternal relationship status were significant for any of the reported analyses, all $p \mathrm{~s}>0.614$.
}

Table 3 Adjusted Means and Standard Errors for the Number of Severe Life Events and Chronic Adversities: Anxious and Control Children

\begin{tabular}{llll}
\hline \multirow{2}{*}{ Rater } & Variable & \multicolumn{2}{l}{ Adjusted mean (SE) } \\
\cline { 3 - 4 } & & Anxious & Controls \\
\hline Mother & Severe life events & $0.97(1.01)$ & $0.38(0.54)$ \\
Panel & Severe life events & $1.33(1.42)$ & $0.97(0.93)$ \\
& Chronic adversities & $2.44(1.88)$ & $1.64(1.67)$ \\
\hline
\end{tabular}

\section{Discussion}

The prediction that anxious children would experience a significantly greater number of severe life events and chronic adversities in the 12 months prior to the onset of their most recent clinical episode than controls was supported. These results are consistent with past research that also utilized an investigator-based interview to assess severe life events and a range of specific chronic adversities preceding the most recent onset of anxiety in children (Goodyer et al. 1988, 1990). However, as past research did not include maternal ratings, comparisons cannot be made regarding the number of severe life events calculated on the basis of maternal perceptions. Importantly, the significant finding for severe life events did not vary as a function of whether impact ratings were provided by mothers or a panel of independent raters. Results supported the presence of a positive linear relationship between current child anxiety symptoms assessed on the basis of maternal and child selfreport and chronic adversities (but not severe life events) prior to onset in the clinical sample. It may be that severe life events are important in the provocation of onset, but are not associated with symptomatology once problems have started. In contrast chronic adversities may reflect stable characteristics of the child, their family or the broader social context (e.g., health problems, discrimination). Indeed, Sandberg et al. (1993) found that approximately $80 \%$ of chronic adversities lasted at least a year in a sample of children referred to psychiatric clinics. Alternatively, the occurrence of chronic adversities may produce long-term changes that increase the child's vulnerability regardless of whether or not the stressor remains present (e.g., parental unemployment results in financial difficulties that persist even after the parent finds a new job).

The hypothesis that more severe life events would be assessed for the anxious sample on the basis of maternal ratings of impact in comparison to panel ratings was not supported. To the contrary, the panel assessed a significantly greater number of severe life events for both groups compared to mothers. Therefore it is unlikely that current findings were the product of a maternal perception bias inflating the number of events. However, it could be argued 
that the assessment of stressors may still have been coloured by a perception bias given that panel ratings were based on information derived from interviews with mothers. One way to have further minimized maternal bias would have been to include the child version of PACE to produce a "best estimate" of the number of events. However, in this study maternal report was considered optimal due to the young age of children, considering that participants were required to recall events over a 12-month time period, and also the mothers' greater ability to provide information about certain areas (e.g., family finances).

Our clinical sample consisted chiefly of children for whom the current episode was a repeat episode of anxiety, with only seven children identified as initial onset cases. This is somewhat unsurprising given that anxiety disorders typically occur within a context of temperamental vulnerability and a history of long-term difficulties with anxiety (Hudson and Rapee 2004; Pérez-Edgar and Fox 2005; Rapee 2001). Therefore current findings are applicable to the triggering of a repeat onset of anxiety in children who have a previous history of anxiety difficulties. ${ }^{2}$ Whether severe life events and chronic adversities are also involved in the initial onset of anxiety is a question that remains to be examined. Future research should include a larger sample to allow for a comparison of initial and repeat onset cases regarding the contribution of severe life events and chronic adversities to the development and course of anxiety disorders.

An alternative explanation for the present findings is that, because children may have experienced additional episode(s) of anxiety during the 12-month period under study, a higher rate of stressors may have been assessed due to anxiety symptoms or temperamental influences leading children to contribute to the occurrence of stressors through their own behaviour. The current study sought to control for this eventuality by excluding events likely to be dependent on the child's behaviour. However, future studies may wish to assess anxiety symptoms and associated impairment in addition to stressors in order to provide a more detailed examination of the relationship between the course of disorder, chronic adversities and life events. Another limitation of the current study is that the interviewer who administered PACE was not blind to the child's diagnostic status. This knowledge may have influenced how the interviews were conducted. However, there are several reasons to think that this was not the case. First, PACE is a highly structured interview, with set questions, definitions

\footnotetext{
${ }^{2}$ The main analyses for severe life events and chronic adversities were repeated excluding children for whom the current episode was also their initial episode of anxiety $(n=7)$. The between subjects main effect of group remained significant for severe life events $(p=0.037)$ and chronic adversities $(p=0.006)$ despite the exclusion of these participants.
}

of terms and clear guidelines for the inclusion of reported experiences. Furthermore, decisions regarding whether an experience met criteria for a life event or a chronic adversity, in addition to the ratings of contextual impact and event independence were made by an independent rater who was blind to the child's diagnostic status. Although it would have been ideal to have used different interviewers to assess stressors and the timing of onset, it is difficult to envisage how one could interview a mother about a wide range of potentially stressful experiences and their impact on her child without becoming aware of whether the child under discussion was a clinical or control participant.

Another methodological concern, typical of research employing a retrospective design, relates to the accuracy of recollections of events some time in the past. The present study required mothers to recall events that may have taken place at any time from 13 to 26 months prior to their interview. It must be noted that although care was taken during interviews to ensure that reported events occurred within the 12-month period under study, it was often difficult to pinpoint the exact date of event occurrence. Consequently the timing of events over the 12-month period prior to onset could not be reported. In addition to problems associated with the fallibility of human memory, it is possible that rates of recalled as opposed to actual events differed between groups due to the influence of memory and attentional biases associated with anxiety. However, recall of the occurrence of objective events may not be as hampered by memory and mood-related biases as is often assumed (Brewin et al. 1993). Indeed, a recent review by Hardt and Rutter (2004) concluded that the validity of retrospective reports is high when clear definitions are provided, as is the case with standardized interviews such as PACE. The authors also suggest ways to enhance the reliability and validity of the assessment of stressors that occur in childhood, such as the use of additional informants (e.g., fathers, siblings) and comparison of retrospective and prospective reports. Such techniques are likely to be helpful in advancing our knowledge of the relationship between stressors and psychological disorders in children.

The generalizability of the current findings may be limited due to sample characteristics. Families were a socially advantaged group, therefore results may not generalize to anxious children from less privileged backgrounds. However, current findings were consistent with those of Goodyer et al. (1988, 1990), whose sample included children from predominantly working-class backgrounds. It is also possible that children with an anxiety disorder who are presenting for treatment may differ from those recruited from within the general community. However given the scarcity of research examining the frequency of stressors prior to onset of anxiety in children, initial research with children recruited in an 
outpatient setting may assist to further develop hypotheses before commencing more costly investigations in community samples.

It must also be noted that a small number of children $(n=7)$ met criteria for a comorbid non-anxiety diagnosis, including depressive disorders. A larger sample would have enabled these cases to be excluded or for a comparison of "pure" versus "mixed" anxiety groups. However, comorbidity with non-anxiety diagnoses is extremely high among children with an anxiety disorder, with epidemiological studies reporting estimates from 15$60 \%$ for depression (Brady and Kendall 1992) and 16$40 \%$ for disruptive behavior disorders (Anderson et al. 1987; Last et al. 1987). Therefore a "pure" anxiety sample would not provide a realistic representation of the nature of anxiety in children. While it is a part of our routine clinic procedure to question parents about the initial onset, course and presence of anxiety and other psychological disorders prior to the child's current diagnoses during assessment, the inclusion of a formal assessment of lifetime diagnoses was not conducted.

This study sought to overcome limitations from prior studies regarding the conceptualization of onset by utilising a definition based on the presence of anxiety symptoms in addition to significant impairment. However, a simple unambiguous time point where onset begins is likely to be the exception rather than the rule (Sandberg and Rutter 2008). Onset may be gradual, with first symptoms appearing long before evidence of significant impairment resulting from the disorder becomes apparent (Sandberg et al. 2001). Furthermore, impairment is likely to lie on a continuum related to the severity of symptoms. Previous research has noted the difficulty of determining the timing of onsets in child psychopathology, despite detailed and time-consuming efforts to enhance accurate recall (Angold et al. 1996; Sandberg et al. 2001). Anxiety disorders in children tend to take a chronic and persistent course and are often associated with signs of an inhibited temperament, present from an early age (Ost and Treffers 2001). Given the importance of onset in investigating causal factors for psychopathology in general the lack of attention paid to its definition and assessment is very surprising. Therefore one difficult yet interesting issue that warrants further discussion and investigation by researchers is how to best determine the cut-off between temperament and disorder.

The current study provides support for increased rates of discrete and chronic stressors prior to the onset of anxiety compared to controls. Several authors have noted that discrete and chronic stressors may have different implications for the development and course of anxiety and other psychological disorders in children. For example, Rutter and Sandberg (1992) suggested that chronic adversities carry a greater risk for overall susceptibility to the development of disorder, whereas discrete life events may provoke the onset of disorder. A similar suggestion has been made that chronic adversity in childhood may predict the early initial onset of anxiety and chronic anxiety disorders, whereas episodic disorders (e.g., depression) are more likely to be triggered by severe life events (Phillips et al. 2005). These speculations could be investigated using a prospective design where children are followed through to early adulthood to cover the period of greatest risk for the initial onset of depression. Furthermore, casecontrol comparisons revealing higher rates of life events and chronic adversities prior to onset in children referred for the treatment of depression or for a range of other problems (e.g., Goodyer et al. 1988, 1990; Sandberg et al. 2001) suggest that stressors may be a function of psychopathology in children generally, as opposed to specific markers of risk for anxiety. Therefore a prospective design extending across the at-risk period as suggested above would also enable an investigation of the specificity of the relationship between the two types of stressors and anxiety, depression and mixed anxiety/ depression. Current theoretical models of child anxiety (Chorpita and Barlow 1998; Rapee 2001; Hudson and Rapee 2004) would benefit by incorporating the distinction between discrete and chronic stressors to guide research seeking to examine their influence on the onset and persistence of internalizing disorders.

The present findings add to the converging evidence linking increased rates of stressors with child anxiety. However, an ongoing debate concerns whether these stressors represent causal factors or if they are simply markers of other causal features and/or processes. Some authors have argued (Rutter and Sandberg 1992; Sandberg et al. 2001) that research must demonstrate that the rate of severe life events and chronic adversities is significantly higher prior to onset than in subsequent time periods in the clinical sample in order to demonstrate a causal link. Sandberg et al. (2001) included within-individual comparisons of the pre-onset period with other time periods in a sample of children referred to psychiatrics clinic and found weak inconclusive time effects, while still showing a casecontrol difference for the pre-onset period. This suggests that it is possible that greater levels of adverse experiences are a constant presence in the lives of emotionally disturbed children, and therefore do not actually trigger the onset of clinical episodes. Therefore one suggested improvement would be the inclusion of an assessment of adverse experiences during an equivalent time period after the most recent onset to ascertain whether rates of life events and chronic adversities differ before and after this date. This would provide a more stringent test of the possible causal link between stressors and the onset of clinical episodes in anxious children. 
The processes influencing the initial onset and recurrence of anxiety are likely to involve a complex mix of risk and protective factors, one of which may include childhood adversity. Current models have identified several factors in addition to external events that promote anxiety, including genetic vulnerability, cognitive biases, and parental modelling and reinforcement of anxious behaviours (cf. Chorpita and Barlow 1998; Hudson and Rapee 2004; Rapee 2001). For example, children born with an anxious temperament may be more likely to perceive stressors as uncontrollable, a cognitive process that may be facilitated by parental modelling and/or encouragement of avoidant coping strategies. This may lead to the formation of a cognitive bias where children are more likely to interpret future events as uncontrollable, amplifying the expression of anxiety. Therefore lower levels of exposure to adversity may be needed to precipitate future episodes given the child's heightened vulnerability. Indeed, Espejo et al. (2007) speculated that childhood anxiety disorders and a "sensitization" process to future stressors may be linked by a common mechanism, such as cognitive and/or biological changes occurring as a consequence of childhood stressor exposure. Further examination of inter-relationships between the factors predicted to mediate and/or moderate the association between stressors and anxiety in children is likely to be a fruitful area for future research.

This study was the first to examine the number of severe life events and chronic adversities in children with a DSMIV diagnosis of anxiety prior to the onset of their most recent episode, including both maternal (subjective) and investigator-based (contextual) life event impact ratings. While this study provides support for the presence of higher rates of severe life events and chronic adversities in anxious children compared to controls, the question as to whether these variables represent causal factors that precipitate onset or whether they simply represent correlates of anxiety disorders in children remains unanswered. However, current findings provide more convincing evidence for a link between onset, life events and chronic adversities due to strengths in methodology than do previous studies through the inclusion of interview assessment of stressors, maternal and independent impact ratings, and an impairment-based definition of onset. Now that this link has been more clearly established, researchers are presented with a firmer base from which to investigate the potential causal role of stressors in precipitating the onset of episodes of anxiety in childhood and potential mediators/moderators of this relationship. For example, elaboration on the possible link between sensitization or enhanced vulnerability to stress and childhood anxiety offers promising possibilities for stress research and for etiological models of child anxiety alike. The findings of the present study indicate that the identification of children at-risk due to exposure to external stressors, and preventative efforts targeting such children, may assist in preventing a persistent course of anxiety.

Acknowledgment We thank Alan Taylor from the Psychology Department, Macquarie University and Andrea Meyer, Institute of Psychology, University of Basel for their assistance with statistical analyses. This study was supported in part by funding from the Australian Rotary Health Research Fund. Jennifer Allen is now at the University of Basel, Switzerland.

\section{References}

American Psychiatric Association (1994). Diagnostic and statistical manual of mental disorders (4th ed.). Washington, DC: American Psychiatric Association.

Anderson, J. C., Williams, S., McGee, R., \& Silva, P. A. (1987). DSM-III disorders in preadolescent children: Prevalence in a large sample from the general population. Archives of General Psychiatry, 44, 69-76.

Angold, A., Erklani, A., Costello, E. J., \& Rutter, M. (1996). Precision, reliability and accuracy in the dating of symptom onsets in child and adolescent psychopathology. Journal of Child Psychology and Psychiatry, 37, 657-664.

Blazer, D., Hughes, D., \& George, L. K. (1987). Stressful life events and the onset of a generalized anxiety syndrome. American Journal of Psychiatry, 144(9), 1178-1183.

Brady, E. U., \& Kendall, P. C. (1992). Comorbidity of anxiety and depression in children and adolescents. Psychological Bulletin, 111(2), 244-255.

Brewin, C. R., Andrews, B., \& Gotlib, I. H. (1993). Psychopathology and early experience: A reappraisal of retrospective reports. Psychological Bulletin, 113, 82-98.

Boer, F., Markus, M. T., Maingay, R., Lindhout, I. E., Borst, S. R., \& Hoogendijk, T. (2002). Negative life events of anxiety disordered children: Bad fortune, vulnerability or reporter bias. Child Psychiatry and Human Development, 32(3), 187-199.

Brown, G. W., \& Harris, T. O. (1978). Social origins of depression. London: Free.

Chorpita, B. F., \& Barlow, D. H. (1998). The development of anxiety: The role of control in the early environment. Psychological Bulletin, 124(1), 3-21.

Eley, T. C., \& Stevenson, J. (2000). Specific life events and chronic experiences differentially associated with depression and anxiety in young twins. Journal of Abnormal Child Psychology, 28(4), 383-394.

Espejo, E. P., Hammen, C. L., Connolly, N., Brennan, P. A., Najman, J. M., \& Bor, W. (2007). Stress sensitization and adolescent depressive severity as a function of childhood adversity: A link to anxiety disorders. Journal of Abnormal Child Psychology, 35(2), 287-289.

Faravelli, C. (1985). Life events preceding the onset of panic disorder. Journal of Affective Disorders, 9, 103-105.

Finlay-Jones, R., \& Brown, G. W. (1981). Types of stressful life events and the onset of anxiety and depressive disorders. Psychological Medicine, 11, 803-815.

Glen, S., Simpson, A., Drinnan, D., McGuinness, D., \& Sandberg, S. (1993). Testing the reliability of a new measure of life events and experiences in childhood: The psychosocial assessment of childhood experiences (PACE). European Child and Adolescent Psychiatry, 2(2), 98-110.

Goodman, R. (1997). The Strengths and Difficulties Questionnaire: A research note. Journal of Child Psychology and Psychiatry, 38 (5), 581-586. 
Goodman, R. (2001). Psychometric properties of the Strengths and Difficulties Questionnaire. Journal of the American Academy of Child and Adolescent Psychiatry, 40(11), 1337-1345.

Goodyer, I. M. (2001). Life events: Their nature and effects. In I. M. Goodyer (Ed.), The depressed child and adolescent (pp. 204232, 2nd ed.). London: Cambridge University Press.

Goodyer, I. M., Wright, C., \& Altham, P. (1988). Maternal adversity and recent stressful life events in anxious and depressed children. Journal of Child Psychology and Psychiatry, 29, 651-667.

Goodyer, I., Wright, C., \& Altham, P. (1990). The friendships and recent life events of anxious and depressed school age children. British Journal of Psychiatry, 156, 689-698.

Gothelf, D., Aharonovsky, O., Horesh, N., Carty, T., \& Apter, A. (2004). Life events and personality factors in children and adolescents with obsessive-compulsive disorder and other anxiety disorders. Comprehensive Psychiatry, 45(3), 192-198.

Hardt, J., \& Rutter, M. (2004). Validity of adult retrospective reports of adverse childhood experiences: A review of the evidence. Journal of Child Psychology and Psychiatry, 45, 260-273.

Hudson, J. L., \& Rapee, R. M. (2004). From anxious temperament to disorder: An etiological model of generalized anxiety disorder. In R. G. Heimberg, C. L. Turk, \& D. S. Mennin (Eds.), The etiology and development of generalised anxiety disorder (pp. 51-74). New York: Guilford.

Last, C. G., Strauss, C. C., \& Francis, G. (1987). Comorbidity among childhood anxiety disorders. Journal of Nervous and Mental Disease, 175(12), 726-730.

Lyneham, H. J., Abbott, M. J., \& Rapee, R. M. (2007). Inter-rater reliability of the Anxiety Disorders Interview Schedule for DSMIV: Child and Parent Version. Journal of the American Academy of Child and Adolescent Psychiatry, 46(6), 731-736.

Nauta, M. H., Scholing, A., Rapee, R. M., Abbott, M., Spence, S. H., \& Waters, A. (2004). A parent-report measure of children's anxiety: Psychometric properties and comparison with childreport in a clinic and normal sample. Behaviour Research and Therapy, 42(7), 813-839.

Ost, L. G., \& Treffers, P. D. A. (2001). Onset, course, and outcome for anxiety disorders in children. In W. K. Silverman, \& P. D. A. Treffers (Eds.), Anxiety disorders in children and adolescents: Research, assessment and intervention (pp. 293-312). New York: Cambridge University Press.

Pérez-Edgar, K., \& Fox, N. A. (2005). Temperament and anxiety disorders. Child and Adolescent Psychiatric Clinics of North America, 14, 681-706.

Phillips, N. K., Hammen, C. L., Brennan, P. A., Najman, J. M., \& Bor, W. (2005). Early adversity and the prospective prediction of depressive and anxiety disorders in adolescents. Journal of Abnormal Child Psychology, 33(1), 13-24.

Rapee, R. M. (2001). The development of generalized anxiety. In M. W. Vasey, \& M. R. Dadds (Eds.), The developmental psychopa- thology of anxiety (pp. 481-503). Oxford: Oxford University Press.

Rapee, R. M., \& Bryant, R. A. (2008). Stress and psychosocial factors in the onset of fear circuitry disorders. In G. Andrews, D. S. Charney, P. Sirovatka, \& R. D. Washington (Eds.), Stress-induced and Fear-Circuitry Disorders. American Psychiatric Press Inc, (in press).

Rapee, R. M., Litwin, E. M., \& Barlow, D. H. (1990). Impact of life events on subjects with panic disorder and on comparison subjects. American Journal of Psychiatry, 147(5), 640-644.

Rapee, R. M., \& Spence, S. H. (2004). The etiology of social phobia: Empirical evidence and an initial model. Clinical Psychology Review, 24, 737-767.

Rapee, R. M., \& Szollos, A. A. (2002). Developmental antecedents of clinical anxiety in childhood. Behaviour Change, 19(3), 146-157.

Roy-Byrne, P. P., Geraci, M., \& Uhde, T. W. (1986). Life events and the onset of panic disorder. American Journal of Psychiatry, 143, 1424-1427.

Rutter, M., \& Sandberg, S. (1992). Psychosocial stressors: Concepts, causes and effects. European Child and Adolescent Psychiatry, 1 (1), 3-13.

Sandberg, S., McGuinness, D., Hillary, C., \& Rutter, M. (1998). Independence of childhood life events and chronic adversities: A comparison of two patient groups and controls. Journal of the American Academy of Child and Adolescent Psychiatry, 37(7), 728-735.

Sandberg, S., \& Rutter, M. (2008). Acute life stresses. In M. Rutter, D. Bishop, D. Pine, S. Scott, J. Stevenson, E. Taylor, \& A. Thapar (Eds.), Rutter's Child and Adolescent Psychiatry (pp. 394-408, 5th ed.). New York: Wiley-Blackwell.

Sandberg, S., Rutter, M., Giles, S., Owen, A., Champion, L., Nicholls, J., et al. (1993). Assessment of psychosocial experiences in childhood: Methodological issues and some illustrative findings. Journal of Child Psychology and Psychiatry, 34(6), 879-897.

Sandberg, S., Rutter, M., Pickles, A., McGuinness, D., \& Angold, A. (2001). Do high-threat life events really provoke the onset of psychiatric disorder in children? Journal of Child Psychology and Psychiatry, 42(4), 523-532.

Silverman, W. K., \& Albano, A. M. (1996). Anxiety disorders interview schedule for DSM-IV: Child and parent versions. San Antonio: The Psychological Corporation.

Silverman, W. K., Saavedra, L. M., \& Pina, A. A. (2001). Test-retest reliability of anxiety symptoms and diagnoses with anxiety disorders interview schedule for DSM-IV: Child and parent versions. Journal of the American Academy of Child and Adolescent Psychiatry, 40 (8), 937-944.

Spence, S. H. (1998). A measure of anxiety symptoms among children. Behaviour Research and Therapy, 36(5), 545-566.

Spence, S. H. (1999). Spence Children's Anxiety Scale (parent version). Brisbane: University of Queensland. 